\title{
Stability of systems with fast-varying delay using improved Wirtinger's inequality
}

\author{
Alexandre Seuret ${ }^{1,2}$, Frédéric Gouaisbaut ${ }^{1,3}$ and Emilia Fridman ${ }^{4}$
}

\begin{abstract}
This paper considers the stability of systems with fast-varying delay. The novelty of the paper comes from the consideration of a new integral inequality which is proved to be less conservative than the celebrated Jensen's inequality. Based on this new inequality, a dedicated construction of LyapunovKrasovskii functionals is proposed and is showed to have a great potential efficient in practice. The method is also combined with an efficient representation of the improved reciprocally convex combination inequality, recently provided in the literature, in order to reduce the conservatism induced by the LMIs optimization setup. The effectiveness of the proposed result is illustrated by some classical examples from the literature.
\end{abstract}

\section{INTRODUCTION}

Time-delay systems arise in many practical situations like in biology, economy or mechanical engineering (see for instance [8], [18], [16], [22]) and references therein). It has motivated a huge number of results devoted to the development of criteria dedicated, more espetially to the stability analysis of linear time-delay systems.

In the case of a constant delay and nominal system, some theoretical tools like direct eigenvalues analysis [22] are now well established and allow to derive efficient criteria with a relatively low numerical complexity. However this type of criteria generally fails in assessing stability for uncertain systems and, more importantly, when the delay becomes time varying.

When the delay is time-varying, two different methodologies have been employed. In the first framework, we aim at transforming the original system into a closed loop between a nominal LTI system and a system depending on the delay. This last element is embedded into an uncertainty and the use of classical tools like Small Gain Theorem [7], [15], IQCs [12] or Quadratic Separation [2] allows to conclude on the stability. Another technique is based on the construction of Lyapunov-Krasovskii functionals (see for instance [5] in order to apply extension of Lyapunov theory to the case of delay systems. The construction of these functionals often induces an important conservatism and several attempts have been proposed to reduce it by choosing extended state based Lyapunov-Krasovskii functionals ([1], [13]), or discretized Lyapunov functionals ([8]). The third step which brings an important source of conservatism comes from the inequalities to be used to derive a tractable numerical optimization problem (see [10], [11], [14], [17], [21], [23]. Generally,

\footnotetext{
${ }^{1}$ CNRS, LAAS, 7 avenue du Colonel Roche, 31077 Toulouse, France. aseuret, fgouaisblaas. fr

2 Univ de Toulouse, LAAS, F-31400 Toulouse, France.

3 Univ de Toulouse, UPS, LAAS, F-31400, Toulouse, France.

${ }^{4}$ Tel Aviv University, Tel Aviv, Israel.
}

all the papers proceed in two stages: Firstly, all the cross terms of the derivative of $V$ are bounded using a Jensen's like inequality. Then, in order to get an LMI with respect to the delay $h(t)$ and the delay derivative $\dot{h}(t)$, the result is transformed into a convex optimization problem often with the help of slack variables [17]. The notion of fastvarying delays appeared in [5] and refers to the case when the stability condition does not depend on the derivative of the delay function $\dot{h}$. In this situation several articles refines the stability conditions [11], [21], [17], [6].

In this paper, unlike many papers in the literature, we focus on the two last stages of the procedure, the development of a less restrictive inequality and the choice of slack variables. First of all, following the work of [14] or [3], we propose a relevant integral inequality which was introduced in [20]. The resulting inequality depends on $x(t), x(t-h(t)$ and also on the integral of the state over a delay interval. This new signal is then directly integrated into a suitable classical Lyapunov function, highlighting so the features of this new inequality. Then, we propose to extend the work of [17] in order to get tractable optimization scheme but with a fewer numbers of slack variables to be optimized.

The paper is organized as follows. Section 2 introduces a new integral inequality. We also propose a new representation of the improved reciprocally convex combination inequality in order to get some tractable convex optimization problem to deal with. Section 3 presents the main result of the paper on the stability analysis of systems with fast varying delays using classical Lyapunov-Krasovskii functional combined with inequalities describes in Section 2. Section 4 illustrates our results with some examples extracted from the literature.

Notations: Throughout the paper $\mathbb{R}^{n}$ denotes the $n$ dimensional Euclidean space with vector norm $|\cdot|, \mathbb{R}^{n \times m}$ is the set of all $n \times m$ real matrices. For any symmetric matrix $P \in \mathbb{R}^{n \times n}$, the notation $P \succ 0$ (or $P \prec 0$ ) means that $P$ is positive (or negative) definite. The set $\mathbb{S}_{n}^{+}$refers to the set of symmetric positive definite matrices. The notation $\left[\begin{array}{cc}A & B \\ * & C\end{array}\right]$ stands for the symmetric matrix $\left[\begin{array}{cc}A & B \\ B^{T} & C\end{array}\right]$. For any matrices $A, B$ in $\mathbb{R}^{n \times n}$, the notation $\operatorname{diag}(A, B)$ denotes the bloc diagonal matrix $\left[\begin{array}{cc}A & 0 \\ * & B\end{array}\right]$.

\section{RELEVANT INEQUALITIES}

In the context of the stability analysis of time delay systems using Lyapunov-Krasovskii functionals, diverse types of functionals have been provided. It has been shown in [4] that 
one of the most relevant term corresponds to the following integral quadratic term

$$
V\left(x_{t}\right)=\int_{t-h}^{t} \int_{\theta}^{t} \dot{x}^{T}(\theta) R \dot{x}(\theta) \mathrm{d} \theta \mathrm{d} s,
$$

where $x$ represents the state of a time-delay systems, and $h>0$ the delay. In order to ensure the positivity of this term, the matrix $R$ is chosen symmetric positive definite. This class of Lyapunov-Krasovskii terms has been widely used in the literature mainly because the computation of its time derivative leads to conditions which depends on the explicit value of the delay $h$. Indeed when differentiating this term with respect to the time variable $t$, we get

$$
\dot{V}\left(x_{t}\right)=h \dot{x}^{T}(t) R \dot{x}(t)-\int_{t-h}^{t} \dot{x}^{T}(s) R \dot{x}(s) \mathrm{d} s .
$$

In order to transcribe this expression in the LMI representation, the trick is to apply the Jensen's inequality . In this article, we aim at presenting a new integral inequality which will be employed in order to derive stability conditions for linear systems with fast-varying delays. More specifically, we formulate a new inequality which is proved to be less conservative compared to Jensen's inequality. Furthermore, based on a reciprocally convex combination lemma provided in [17], we propose some new relaxations for this lemma which allows to reduce the number of variables involved in the optimization setup.

\section{A. Improved integral inequality}

The following lemma provides a new integral inequality which is less conservative than the well-known Jensen's inequality and than the inequality derived from the Wirtinger's inequality given in [19], [20].

Lemma 2.1: For a given symmetric positive definite matrix $R \in \mathbb{S}_{n}^{+}$, any differentiable function $\omega$ in $[a, b] \rightarrow \mathbb{R}^{n}$, then the following inequality holds:

$$
\int_{a}^{b} \dot{\omega}(u) R \dot{\omega}(u) \mathrm{d} u \geq \frac{1}{b-a}\left[\begin{array}{l}
\Omega_{0} \\
\Omega_{1}
\end{array}\right]^{T} \tilde{R}\left[\begin{array}{l}
\Omega_{0} \\
\Omega_{1}
\end{array}\right],
$$

where

$$
\begin{aligned}
\Omega_{0} & =\omega(b)-\omega(a), \\
\Omega_{1} & =\omega(b)+\omega(a)-\frac{2}{b-a} \int_{a}^{b} \omega(u) \mathrm{d} u, \\
\tilde{R} & =\operatorname{diag}(R, 3 R) .
\end{aligned}
$$

Proof. For any sufficiently differentiable function $\omega$ in $[a, b] \rightarrow \mathbb{R}^{n}$, consider a signal $z$ given, for all $u \in[a, b]$ by

$$
z(u)=\dot{\omega}(u)-\frac{1}{b-a} \Omega_{0}+3 \frac{(b+a-2 u)}{(b-a)^{2}} \Omega_{1},
$$

where $\Omega$ has been defined in the statements of the lemma. The computation of $\int_{a}^{b} z^{T}(u) R z(u) \mathrm{d} u$ leads to:

$$
\begin{aligned}
\int_{a}^{b} z^{T}(u) R z(u) \mathrm{d} u & =\int_{a}^{b} \dot{\omega}^{T}(u) R \dot{\omega}(u) \mathrm{d} u+\frac{\int_{a}^{b} 1 \mathrm{~d} u}{(b-a)^{2}} \Omega_{0}^{T} R \Omega_{0} \\
& +9 \frac{\int_{a}^{b}(b+a-2 u)^{2} \mathrm{~d} u}{(b-a)^{4}} \Omega_{1}^{T} R \Omega_{1} \\
& -\frac{2}{b-a} \int_{a}^{b} \dot{\omega}^{T}(u) \mathrm{d} u R \Omega_{0} \\
& +\frac{6}{(b-a)^{2}} \int_{a}^{b}(b+a-2 u) \dot{\omega}^{T}(u) \mathrm{d} u R \Omega_{1} \\
& -\frac{6}{(b-a)^{3}} \int_{a}^{b}(b+a-2 u) \mathrm{d} u \Omega_{0}^{T} R \Omega_{1} .
\end{aligned}
$$

Simple calculus ensure that

$$
\begin{aligned}
\int_{a}^{b}(b+a-2 u)^{2} \mathrm{~d} u & =\frac{1}{3}(b-a)^{3}, \\
\int_{a}^{b}(b+a-2 u) \mathrm{d} u & =0 \\
\int_{a}^{b} \dot{\omega}(u) \mathrm{d} u & =\Omega_{0},
\end{aligned}
$$

and an integration by parts ensures that

$$
\int_{a}^{b}(b+a-2 u) \dot{\omega}^{T}(u) \mathrm{d} u=-2(b-a) \Omega_{1} .
$$

It thus follows that

$$
\begin{aligned}
\int_{a}^{b} z^{T}(u) R z(u) \mathrm{d} u & =\int_{a}^{b} \dot{\omega}^{T}(u) R \dot{\omega}(u) \mathrm{d} u \\
& +\frac{1-2}{(b-a)} \Omega_{0}^{T} R \Omega_{0} \\
& +\frac{9-12}{(b-a)} \Omega_{1}^{T} R \Omega_{1} .
\end{aligned}
$$

or equivalently

$$
\begin{aligned}
\int_{a}^{b} z^{T}(u) R z(u) \mathrm{d} u & =\int_{a}^{b} \dot{\omega}^{T}(u) R \dot{\omega}(u) \mathrm{d} u \\
& -\frac{1}{b-a}\left[\begin{array}{c}
\Omega_{0} \\
\Omega_{1}
\end{array}\right]^{T}\left[\begin{array}{cc}
R & 0 \\
0 & 3 R
\end{array}\right]\left[\begin{array}{l}
\Omega_{0} \\
\Omega_{1}
\end{array}\right] .
\end{aligned}
$$

The proof is concluded by noting that the left hand side of the previous equality is positive definite since $R \succ 0$.

Remark 1: The differences between the previous inequality and the Jensen's inequality are the following: in Lemma 2.1 , the term $\frac{1}{b-a} \Omega_{0}^{T} R \Omega_{0}$ refers exactly to the right hand side of the Jensen's inequality. Then the term $\frac{3}{b-a} \Omega_{1}^{T} R \Omega_{1}$ is non-negative. Thus it is clear that this new inequality encompasses the Jensen's inequality. It is also worth noting that this improvement is allowed by using an extra signal $\int_{a}^{b} \omega(u) \mathrm{d} u$ and not only the signals $\omega(b)$ and $\omega(a)$. Therefore, it suggests that in order to be useful, this inequality should be combined with a Lyapunov functional where the signal $\int_{a}^{b} \omega(u) \mathrm{d} u$ appears explicitly.

Remark 2: A similar integral inequality based on Wirtinger's inequality has been recently proposed in [19]. The difference with respect to this inequality relies in the coefficient 3 which replaces the previously obtained coefficient $\pi^{2} / 4$ (which is lower than 3). Thus, this new approach leads to a less conservative result.

\section{B. Improved reciprocally convex combination inequality}

The following is largely inspired from the reciprocally convex combination lemma provided in [17].

Lemma 2.2: Let $n, m$ be two positive integers, and two matrices $R_{1}$ in $\mathbb{S}_{n}^{+}$and $R_{2}$ in $\mathbb{S}_{m}^{+}$. 
Consider a scalar $\alpha$ in the interval $(0,1)$ and introduce the matrix $\Theta(\alpha)$ as:

$$
\Theta(\alpha)=\left[\begin{array}{cc}
\frac{1}{\alpha} R_{1} & 0 \\
* & \frac{1}{1-\alpha} R_{2}
\end{array}\right],
$$

The improved reciprocally convex combination guarantees that, if there exists a matrix $X$ in $\mathbb{R}^{n \times m}$ such that

$$
\Theta_{m}=\left[\begin{array}{cc}
R_{1} & X \\
* & R_{2}
\end{array}\right] \succeq 0,
$$

then the following inequality holds

$$
\Theta(\alpha) \succeq \Theta_{m}
$$

Proof. The proof is largely inspired from [17]. Define the functions $f_{i j}$ for $i, j=1,2,3$ and for all $\alpha \in\left(\begin{array}{ll}0 & 1\end{array}\right)$ as follows.

$$
f_{12}(\alpha)=f_{21}^{-1}(\alpha)=\frac{\sqrt{1-\alpha}}{\sqrt{\alpha}} .
$$

In order to simplify the notations, the arguments of the function $f_{i j}$ will be omitted. Let $e_{1}$ and $e_{2}$ be two vectors of $\mathbb{R}^{n}$ and $\mathbb{R}^{m}$, respectively. Define the function $\tilde{\Theta}$ by

$$
\begin{aligned}
\tilde{\Theta}(\alpha) & =\left[\begin{array}{c}
e_{1} \\
e_{2}
\end{array}\right]^{T}\left[\begin{array}{cc}
R_{1} & X \\
* & R_{2}
\end{array}\right]\left[\begin{array}{l}
e_{1} \\
e_{2}
\end{array}\right] \\
& +\left[\begin{array}{c}
f_{12} e_{1} \\
-f_{21} e_{2}
\end{array}\right]^{T}\left[\begin{array}{cc}
R_{1} & X \\
* & R_{2}
\end{array}\right]\left[\begin{array}{c}
f_{12} e_{1} \\
-f_{21} e_{2}
\end{array}\right] .
\end{aligned}
$$

Some computations shows that:

$$
\begin{aligned}
\tilde{\Theta}(\alpha) & =\left(1+f_{12}^{2}\right) e_{1}^{T} R_{1} e_{1} \\
& +\left(1+f_{21}^{2}\right) e_{2}^{T} R_{2} e_{2} \\
& +2\left(1-f_{12} f_{21}\right) e_{1}^{T} X e_{2} .
\end{aligned}
$$

From the definition of the functions $f_{12}$ and $f_{21}$, it holds $1+f_{12}^{2}=1 / \alpha, 1+f_{21}^{2}=1 /(1-\alpha)$ and $f_{12} f_{21}=1$. This implies

$$
\tilde{\Theta}(\alpha)=\left[\begin{array}{l}
e_{1} \\
e_{2}
\end{array}\right]^{T} \Theta(\alpha)\left[\begin{array}{l}
e_{1} \\
e_{2}
\end{array}\right] .
$$

Since the condition $\Theta_{m} \succeq 0$ holds, the last term of $\tilde{\Theta}$ is positive definite which ensures

$$
\left[\begin{array}{l}
e_{1} \\
e_{2}
\end{array}\right]^{T}\left(\Theta(\alpha)-\Theta_{m}\right)\left[\begin{array}{l}
e_{1} \\
e_{2}
\end{array}\right] \geq 0
$$

This inequality holds for all vectors $e_{1}$ and $e_{2}$ and is equivalent to

$$
\Theta(\alpha)-\Theta_{m} \succeq 0,
$$

which concludes the proof.

Remark 3: The previous lemma gives another interpretation of the improved reciprocally convex combination lemma provided in [17]. This new formulation provides a new matrix inequality which can be directly applied to a stability analysis of time-delay systems.

In the sequel, the two previous lemmas will be employed in order to derive stability conditions for linear systems with fast-varying delays.

\section{STABILITY ANALYSIS OF TIME-VARYING DELAY SYSTEMS}

This section exposes a novel stability theorem for systems with fast-varying delay, which is based on the use of the two previous lemmas. Consider a linear time-delay system of the form:

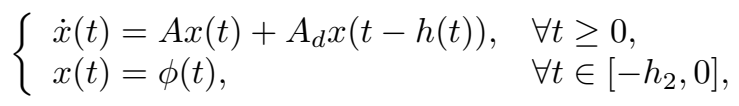

where $x(t) \in \mathbb{R}^{n}$ is the state vector, $\phi$ is the initial condition and $A, A_{d} \in \mathbb{R}^{n \times n}$ are constant matrices.

The delay is assumed to be time-varying. The classical constraints on the delay function are

$$
h(t) \in\left[h_{1}, h_{2}\right], \quad \forall t \geq 0,
$$

where $h_{1} \leq h_{2}$ are positive scalars. Notet that no assumption on the derivative of the delay function are made.

Based on the previous inequality and classical results on Lyapunov-Krasovskii functionals, the stability theorem is provided.

Theorem 1: Assume that there exist matrices $P$ in $\mathbb{S}_{+}^{3 n}, S$, $R$ in $\mathbb{S}_{+}^{n}$, and a matrix $X$ in $\mathbb{R}^{2 n \times 2 n}$. such that the following LMIs are satisfied for $h$ in $\left\{h_{1}, h_{2}\right\}$

$$
\begin{aligned}
& \Psi \quad=\left[\begin{array}{cc}
\tilde{R}_{2} & X \\
* & \tilde{R}_{2}
\end{array}\right] \quad \succ 0, \\
& \Phi(h)=\Phi_{0}(h)-\Gamma^{T} \Psi \Gamma-G_{2}^{T} \tilde{R}_{1} G_{2} \prec 0,
\end{aligned}
$$

where

$$
\begin{aligned}
\Phi_{0}(h)= & G_{1}^{T}(h) P G_{0}+G_{0}^{T} P G_{1}(h)+\hat{S} \\
& +G_{0}^{T}\left(h_{1}^{2} \hat{R}_{1}+h_{12}^{2} \hat{R}_{2}\right) G_{0}, \\
\hat{S}= & \operatorname{diag}\left(S_{1},-S_{1}+S_{2},-S_{2}, 0_{3 n}\right),
\end{aligned}
$$

and where for $i=1,2$

$$
\begin{aligned}
& \hat{R}_{i}=\operatorname{diag}\left(R_{i}, 0_{2 n}\right), \\
& \tilde{R}_{i}=\operatorname{diag}\left(R_{i}, 3 R_{i}\right), \quad \forall i=1,2,
\end{aligned}
$$

and

$$
\begin{aligned}
& G_{0}=\left[\begin{array}{ccccccc}
A & 0 & A_{d} & 0 & 0 & 0 & 0 \\
I & -I & 0 & 0 & 0 & 0 & 0 \\
0 & I & 0 & -I & 0 & 0 & 0
\end{array}\right] \\
& G_{1}(h)=\left[\begin{array}{ccc}
I & 0 & 0 \\
0 & 0 & 0 \\
0 & 0 & 0 \\
0 & 0 & 0 \\
0 & h_{1} I & 0 \\
0 & 0 & \left(h-h_{1}\right) I \\
0 & 0 & \left(h_{2}-h\right) I
\end{array}\right]^{T} \text {, } \\
& G_{2}=\left[\begin{array}{ccccccc}
I & -I & 0 & 0 & 0 & 0 & 0 \\
I & I & 0 & 0 & -2 I & 0 & 0
\end{array}\right] \text {, } \\
& G_{3}=\left[\begin{array}{ccccccc}
0 & I & -I & 0 & 0 & 0 & 0 \\
0 & I & I & 0 & 0 & -2 I & 0
\end{array}\right] \text {, } \\
& G_{4}=\left[\begin{array}{ccccccc}
0 & 0 & I & -I & 0 & 0 & 0 \\
0 & 0 & I & I & 0 & 0 & -2 I
\end{array}\right] \text {, } \\
& \Gamma=\left[\begin{array}{ll}
G_{3}^{T} & G_{4}^{T}
\end{array}\right]^{T} .
\end{aligned}
$$


Then the system (5) is asymptotically stable for all timevarying delay functions $h$ satisfying (6).

Proof. Consider the Lyapunov-Krasovskii functional given by

$$
V\left(x_{t}, \dot{x}_{t}\right)=V_{1}\left(x_{t}\right)+V_{2}\left(x_{t}\right)+V_{3}\left(x_{t}, \dot{x}_{t}\right),
$$

where

$$
\begin{aligned}
V_{1}\left(x_{t}\right) & =\left[\begin{array}{c}
x(t) \\
\int_{t-h_{1}}^{t} x(s) \mathrm{d} s \\
\int_{t-h_{2}}^{t} x(s) \mathrm{d} s
\end{array}\right]^{T} P\left[\begin{array}{c}
x(t) \\
\int_{t-h_{1}}^{t} x(s) \mathrm{d} s \\
\int_{t-h_{2}}^{t-h_{1}} x(s) \mathrm{d} s
\end{array}\right], \\
V_{2}\left(x_{t}\right) & =\int_{t-h_{1}}^{t} x^{T}(s) S_{1} x(s) \mathrm{d} s \\
& +\int_{t-h_{1}}^{t} x^{T}(s) S_{2} x(s) \mathrm{d} s, \\
V_{3}\left(x_{t}, \dot{x}_{t}\right) & =h_{1} \int_{-h_{1}}^{0} \int_{t+\theta}^{t} \dot{x}^{T}(s) R_{1} \dot{x}(s) \mathrm{d} s \mathrm{~d} \theta \\
& +h_{12} \int_{-h_{2}}^{-h_{1}} \int_{t+\theta}^{t} \dot{x}^{T}(s) R_{2} \dot{x}(s) \mathrm{d} s \mathrm{~d} \theta,
\end{aligned}
$$

where $h_{12}=h_{2}-h_{1}$.

This functional is positive definite since the matrices $P$, $S_{1}, S_{2}, R_{1}$ and $R_{2}$ are symmetric positive definite. The functionals $V_{2}$ and $V_{3}$ are the same as the ones that have been used in [17]. The only difference in the functional comes from the first quadratic term which does not only depend on $x(t)$ but on an augmented vector which includes the additional signals $\int_{t-h_{1}}^{t} x(s) \mathrm{d} s$ and $\int_{t-h_{2}}^{t-h_{1}} x(s) \mathrm{d} s$. This term refers to several functionals that can be found in the literature see for instance [8]. The derivative of the functionals along the trajectories of the system leads to

$$
\dot{V}\left(x_{t}, \dot{x}_{t}\right)=\dot{V}_{1}\left(x_{t}\right)+\dot{V}_{2}\left(x_{t}\right)+\dot{V}_{3}\left(x_{t}, \dot{x}_{t}\right) .
$$

Introduce the augmented vector $\zeta$ as

$$
\zeta(t)=\left[\begin{array}{c}
x(t) \\
x\left(t-h_{1}\right) \\
x(t-h(t)) \\
x\left(t-h_{2}\right) \\
\frac{1}{h_{1}} \int_{t-h_{1}}^{t} x(s) \mathrm{d} s \\
\frac{1}{h(t)-h_{1}} \int_{t-h(t)}^{t-h(t)} x(s) \mathrm{d} s \\
\frac{1}{h_{2}-h(t)} \int_{t-h_{2}}^{t-h(t)} x(s) \mathrm{d} s
\end{array}\right]
$$

Then, by noting that,

$$
\begin{aligned}
\int_{t-h_{2}}^{t-h_{1}} x(s) \mathrm{d} s & =\frac{h(t)-h_{1}}{h(t)-h_{1}} \int_{t-h(t)}^{t-h_{1}} x(s) \mathrm{d} s \\
& +\frac{h_{2}-h(t)}{h_{2}-h(t)} \int_{t-h_{2}}^{t-h(t)} x(s) \mathrm{d} s
\end{aligned}
$$

the following relation is obtained

$$
\left[\begin{array}{c}
x(t) \\
\int_{t-h_{1}}^{t} x(s) \mathrm{d} s \\
\int_{t-h_{2}}^{t-h_{1}} x(s) \mathrm{d} s
\end{array}\right]=G_{1}(h) \zeta(t) .
$$

Moreover it is easy to see that

$$
\begin{aligned}
\frac{\mathrm{d}}{\mathrm{d} t}\left[\begin{array}{c}
x(t) \\
\int_{t-h_{1}}^{t} x(s) \mathrm{d} s \\
\int_{t-h_{2}}^{t-h_{1}} x(s) \mathrm{d} s
\end{array}\right] & =\left[\begin{array}{c}
\dot{x}(t) \\
x(t)-x\left(t-h_{1}\right) \\
x\left(t-h_{1}\right)-\left(t-h_{2}\right)
\end{array}\right] \\
& =G_{0} \zeta(t) .
\end{aligned}
$$

Finally, according to the definition of the vector $\zeta$, the derivative of $V_{1}$ along the trajectories of the system leads to

$$
\dot{V}_{1}\left(x_{t}\right)=\zeta^{T}(t)\left(G_{1}^{T}(h) P G_{0}+G_{0}^{T} P G_{1}(h)\right) \zeta(t) .
$$

According to the definition of the matrix $\tilde{S}$, the derivative of $V_{2}$ along the trajectories of the system yields

$$
\begin{aligned}
\dot{V}_{2}\left(x_{t}\right)= & x^{T}(t) S_{1} x(t)-x\left(t-h_{1}\right)\left(S_{1}-S_{2}\right) x\left(t-h_{1}\right) \\
& -x\left(t-h_{2}\right) S_{2} x\left(t-h_{2}\right) \\
= & \zeta^{T}(t) \tilde{S} \zeta(t) .
\end{aligned}
$$

The derivative of the last term $V_{3}$ along the trajectories of the system leads to

$$
\begin{aligned}
\dot{V}_{3}\left(x_{t}, \dot{x}_{t}\right) & =\dot{x}^{T}(t)\left(h_{1}^{2} R_{1}+h_{12}^{2} R_{2}\right) \dot{x}(t) \\
& -h_{1} \int_{t-h_{1}}^{t} \dot{x}^{T}(s) R_{1} \dot{x}(s) \mathrm{d} s \\
& -h_{12} \int_{t-h_{2}}^{t-h_{1}} \dot{x}^{T}(s) R_{2} \dot{x}(s) \mathrm{d} s .
\end{aligned}
$$

According to the definition of the matrices $G_{0}, \hat{R}_{1}$ and $\hat{R}_{2}$, the previous expression can be rewritten as follows

$$
\begin{aligned}
\dot{V}_{3}\left(x_{t}, \dot{x}_{t}\right) & =\zeta^{T}(t) G_{0}^{T}\left(h_{1}^{2} \hat{R}_{1}+h_{12}^{2} \hat{R}_{2}\right) G_{0} \zeta(t) \\
& -h_{1} \int_{t-h_{1}}^{t} \dot{x}^{T}(s) R_{1} \dot{x}(s) \mathrm{d} s \\
& -h_{12} \int_{t-h_{2}}^{t-h_{1}} \dot{x}^{T}(s) R_{2} \dot{x}(s) \mathrm{d} s .
\end{aligned}
$$

Consider the first integral of the previous equation and applying Lemma 2.1, the following upper bound is obtained according to the definition of the matrix $\tilde{R}_{1}$

$$
\begin{aligned}
& -h_{1} \int_{t-h_{1}}^{t} \dot{x}^{T}(s) R_{1} \dot{x}(s) \mathrm{d} s \leq \\
& -\left[\begin{array}{l}
x(t)-x\left(t-h_{1}\right) \\
x(t)+x\left(t-h_{1}\right)-\frac{2}{h_{1}} \int_{t-h_{1}}^{t} x(s)
\end{array}\right]^{T} \tilde{R}_{1} \\
& \quad \times\left[\begin{array}{l}
x(t)-x\left(t-h_{1}\right) \\
x(t)+x\left(t-h_{1}\right)-\frac{2}{h_{1}} \int_{t-h_{1}}^{t} x(s)
\end{array}\right] .
\end{aligned}
$$

From the definition of the matrix $G_{2}$, the previous expression is rewritten as follows

$$
-h_{1} \int_{t-h_{m}}^{t} \dot{x}^{T}(s) R_{1} \dot{x}(s) \mathrm{d} s \leq-\zeta^{T}(t) G_{2}^{T} \tilde{R}_{1} G_{2} \zeta(t) .
$$

Consider now the last integral term of (13). It holds

$$
\begin{aligned}
\int_{t-h_{2}}^{t-h_{1}} \dot{x}^{T}(s) R_{2} \dot{x}(s) \mathrm{d} s & =\int_{t-h(t)}^{t-h_{1}} \dot{x}^{T}(s) R_{2} \dot{x}(s) \mathrm{d} s \\
& +\int_{t-h_{2}}^{t-h(t)} \dot{x}^{T}(s) R_{2} \dot{x}(s) \mathrm{d} s .
\end{aligned}
$$

and the second integral can be bounded by applying the same procedure as for the previous term

$$
\begin{array}{r}
-h_{12} \int_{t-h_{2}}^{t-h_{1}} \dot{x}^{T}(s) R \dot{x}(s) \mathrm{d} s \leq \\
-\frac{h_{12}}{h(t)-h_{1}} \zeta^{T}(t) G_{3}^{T} \tilde{R}_{2} G_{3} \zeta(t) \\
-\frac{h_{12}}{h_{2}-h(t)} \zeta^{T}(t) G_{4}^{T} \tilde{R}_{2} G_{4} \zeta(t),
\end{array}
$$

which is reformulated in order to apply Lemma 2.2 as

$$
\begin{aligned}
& -h_{12} \int_{t-h_{2}}^{t-h_{1}} \dot{x}^{T}(s) R \dot{x}(s) \mathrm{d} s \leq \\
& \quad-\zeta^{T}(t) \Gamma^{T}\left[\begin{array}{cc}
\frac{h_{12}}{h(t)-h_{1}} \tilde{R}_{2} & 0 \\
* & \frac{h_{12}}{h_{2}-h(t)} \tilde{R}_{2}
\end{array}\right] \Gamma \zeta(t) .
\end{aligned}
$$

Note that

$$
\begin{aligned}
& \lim _{h(t) \rightarrow h_{1}} \frac{G_{3} \zeta(t)}{h(t)-h_{1}}=\left[\begin{array}{c}
\dot{x}^{T}\left(t-h_{1}\right) \\
\dot{x}^{T}\left(t-h_{1}\right)
\end{array}\right], \\
& \lim _{h(t) \rightarrow h_{2}} \frac{G_{4} \zeta(t)}{h_{2}-h(t)}=\left[\begin{array}{c}
\dot{x}^{T}\left(t-h_{2}\right) \\
\dot{x}^{T}\left(t-h_{2}\right)
\end{array}\right] .
\end{aligned}
$$


It implies that the previous inequality is still valid when $h(t)$ tends to $h_{1}$ or $h_{2}$.

Applying Lemma 2.2 with $\alpha=\left(h(t)-h_{1}\right) / h_{12}$, it yields that, if there exists a matrix $X$ in $\mathbb{R}^{2 n \times 2 n}$ such that the matrix $\Psi$ is semi definite positive, then the following inequality holds

$$
-h_{12} \int_{t-h_{2}}^{t-h_{1}} \dot{x}^{T}(s) R_{2} \dot{x}(s) \mathrm{d} s \leq-\zeta^{T}(t) \Gamma^{T} \Psi \Gamma \zeta(t) .
$$

Replacing the upper bounds of the functional $\dot{V}_{1}, \dot{V}_{2}$ and $\dot{V}_{3}$ in (11), it yields

$$
\dot{V}\left(x_{t}, \dot{x}_{t}\right) \leq \zeta^{T}(t) \Phi(h) \zeta(t) .
$$

Then the system is asymptotically stable if the LMI $\Phi(h) \prec 0$ is satisfied for all $h \in\left[h_{1}, h_{2}\right]$. Since $\Phi(h)$ is affine with respect to $h(t)$, a necessary and sufficient condition is to test the LMI only on the vertices of the interval, i.e. $\Phi\left(h_{1}\right) \prec 0$ and $\Phi\left(h_{2}\right) \prec 0$. Then if these two conditions hold, the system is asymptotically stable for all time-varying delay in the interval $\left[h_{1} h_{2}\right]$.

Remark 4: The Lyapunov-Krasovskii functional employed to derive the stability condition of Theorem 1 is similar to the one used in [17] or, more generally, to the ones that can be found in the literature (see for instance [21], [23] among many others). However, a considerable reduction of conservatism comes from the use of the new inequality exposed in Lemma 2.1 as it will be shown in Section IV.

Remark 5: This new integral inequality has been already employed to cope with the problem of time varying delay in [20]. The method was similar but it was also unable to ensure stability for fast varying delays. Indeed the first term of the Lyapunov-Krasovskii functional is given by

$$
V_{1}\left(x_{t}\right)=\left[\begin{array}{c}
x(t) \\
\int_{t-h(t)}^{t} x(s) \mathrm{d} s \\
\int_{t-h_{2}}^{t-h(t)} x(s) \mathrm{d} s
\end{array}\right]^{T} P\left[\begin{array}{c}
x(t) \\
\int_{t-h(t)}^{t} x(s) \mathrm{d} s \\
\int_{t-h_{2}}^{t-h(t)} x(s) \mathrm{d} s
\end{array}\right]
$$

Then, the derivative of the delay function $\dot{h}$ unavoidably appears when differentiating the Lyapunov-Krasovskii functional. Then, there were no possibility to derive stability for systems with fast-varying delays.

\section{EXAMPLES}

The purpose of the following section is to show how the previous theorems can be relevant for the stability analysis of linear systems with fast-varying delays. Especially, we will show that Theorem 1 provides with less conservative results than existing stability conditions from the literature.

\section{A. Example 1}

Consider the following linear time-delay system (5) with:

$$
A=\left[\begin{array}{rr}
-2.0 & 0.0 \\
0.0 & -0.9
\end{array}\right], \quad A_{d}=\left[\begin{array}{rr}
-1.0 & 0.0 \\
-1.0 & -1.0
\end{array}\right] \text {. }
$$

This system is a well-known delay dependent stable system, that is the delay free system is stable and the maximum allowable delay $h_{\max }=6.1725$ can be easily computed by delay sweeping techniques. However this method does not

\begin{tabular}{|c|c|c|c|c|c|c|}
\hline$h_{1}$ & 0.0 & 0.3 & 0.5 & 0.8 & 1.0 & 2.0 \\
\hline$[11]$ & 0.67 & 0.91 & 1.07 & 1.33 & 1.50 & 2.39 \\
{$[9]$} & 0.77 & 0.94 & 1.09 & 1.34 & 1.51 & 2.40 \\
{$[21]$} & 0.87 & 1.07 & 1.21 & 1.45 & 1.61 & 2.47 \\
{$[17]$} & 1.06 & 1.24 & 1.38 & 1.60 & 1.75 & 2.58 \\
\hline Th.1 & 1.51 & 1.62 & 1.70 & 1.88 & 2.02 & 2.81 \\
\hline
\end{tabular}

TABLE II

ADMISSIBLE UPPER BOUND $h_{2}$ FOR VARIOUS $h_{1}$ FOR THE SYSTEM DESCRIBED IN EXAMPLE (15).

provide stability in the case of time-varying delays. We will show that the stability conditions from Theorem 1 is able to assess stability in a more efficient manner than other results from the literature.

The results are summarized in Table I. In [11] or in [21], the stability condition are restricted by the use of the Jensen's inequality. The contribution in [17] provides a notable improvement of the stability conditions due to the use of the improved reciprocally convex combination inequality together with the use of the Jensen's inequality. The results obtained by solving Theorem 1 also show a clear reduction of the conservatism. This is due to the use of both improved reciprocally convex combination inequality and the new integral inequality.

The main benefits of our method is also the obtention of stability region when the delay function is greater than 4.47 which corresponds to the limit values delivered by the Jensen's inequality. Only [6], based on delay partitioning and Theorem 1 are able to ensure stability for delays larger than 4.47 .

The reduction of the conservatism of the stability conditions is at the price of an increasing numerical complexity which is slightly lower in Theorem 1 than in [6].

Of course the cost for reducing the consertism of the stability conditions is the number of variables required in Theorem 1.

\section{B. Example 2}

Let us consider now a second example the following linear time-delay system (5) with:

$$
A=\left[\begin{array}{rr}
0.0 & 1.0 \\
-1.0 & -2.0
\end{array}\right], \quad A_{d}=\left[\begin{array}{rr}
0.0 & 0.0 \\
-1.0 & 1.0
\end{array}\right] \text {. }
$$

This example has been taken from [17]. The results are exposed in Table II. Again Theorem 1 delivers significantly better results than the existing results taken from the litterature.

\section{Example 3}

Theorem 1 addresses also the stability of systems with interval delays, which may be unstable for small delays (or without delays) as it is illustrated with the second example.

$$
A=\left[\begin{array}{rr}
0.0 & 1.0 \\
-2.0 & 0.1
\end{array}\right], \quad A_{d}=\left[\begin{array}{ll}
0.0 & 0.0 \\
1.0 & 0.0
\end{array}\right] \text {. }
$$




\begin{tabular}{|c|c|c|c|c|c|c|c||c||c|}
\hline$h_{1}$ & 0.0 & 1.0 & 2.0 & 3.0 & 4.0 & 5.0 & 6.0 & $h_{1}=h_{2}$ & $\mathrm{~N}$ of variables \\
\hline \hline$[11]$ & 1.01 & 1.64 & 2.39 & 3.20 & 4.06 & - & - & 4.47 & $4 n^{2}+2 n$ \\
{$[21]$} & 1.52 & 1.87 & 2.50 & 3.25 & 4.07 & - & - & 4.47 & $2.5 n^{2}+2.5 n$ \\
{$[17]$} & 1.86 & 2.06 & 2.61 & 3.31 & 4.09 & - & - & 4.47 & $3.5 n^{2}+2.5 n$ \\
{$[6]$} & 1.86 & 2.12 & 2.72 & 3.45 & 4.25 & 5.09 & - & 5.96 & $11.5 n^{2}+3.5 n$ \\
\hline \hline Th.1 & 2.18 & 2.51 & 2.92 & 3.62 & 4.38 & 5.18 & 6.01 & 6.05 & $9.5 n^{2}+2.5 n$ \\
\hline
\end{tabular}

TABLE I

ADMISSIBLE UPPER BOUND $h_{2}$ FOR VARIOUS $h_{1}$ FOR THE SYSTEM DESCRIBED IN EXAMPLE (14).

\begin{tabular}{|c|c|c|c|c|c|c|c|}
\hline$h_{1}$ & 0.11 & 0.3 & 0.5 & 0.8 & 1.0 & 1.3 & 1.5 \\
\hline Th.1 & 0.42 & 1.09 & 1.36 & 1.52 & 1.56 & 1.57 & 1.54 \\
\hline
\end{tabular}

TABLE III

ADMISSIBLE UPPER BOUND $h_{2}$ FOR VARIOUS $h_{1}$ FOR THE SYSTEM DESCRIBED IN EXAMPLE (16).

As $\operatorname{Re}\left(\operatorname{eig}\left(A+A_{d}\right)\right)=0.05>0$, the delay free system is unstable and in this case, the results to assess stability of this system are much more scarce. For the constant delay case, a frequency approach shows that the solutions of this system are stable if the delay belongs to the interval ]0.10017, 1.7178[. It is worth noting that classical LyapunovKrasovskii approaches based on Jensen's inequality cannot assess stability of such systems. However the stability condition of Theorem 1 have solutions thanks to the use of the new integral inequality provided in Lemma 2.1. Only few methods from the literature are able to provide relevant results for this systems. The table shows that Theorem 1 provides stability conditions which are more tight than the ones from the literature.

The results are resumed in Tables III which shows the results. The results obtained by solving the conditions from Theorem 1 with $h_{1}=h_{2}$ leads to stability for all constant delays in the interval $[0.10061 .54]$, which excludes 0 .

\section{Conclusions}

This article presents a new integral inequality which has been proved to be suitable for the stability analysis of systems with fast-varying delays. Combining it with the reciprocal convexity lemma leads to less conservative stability conditions than the usual ones which consider the Lyapunov-Krasovskii approach. It is also shown that for fastvarying delays, the proposed method is less conservative than the ones which are based on IQC's [12]. Furthers research aims at reducing the conservatism with respect to the IQC approach for slow-varying delays.

\section{REFERENCES}

[1] Y. Ariba and F. Gouaisbaut. An augmented model for robust stability analysis of time-varying delay systems. Int. J. Control, 82:1616-1626, September 2009.

[2] Y. Ariba, F. Gouaisbaut, and K.H. Johansson. Robust stability of time-varying delay systems: The quadratic separation approach. Asian Journal of Control, page n/an/a, 2012.
[3] C. Briat. Convergence and equivalence results for the Jensen's inequality - application to time-delay and sampled-data systems. IEEE Transactions on Automatic Control, 56(7):1660-1665, 2011.

[4] E. Fridman and U. Shaked. A descriptor system approach to $H^{\infty}$ control of linear time-delay systems. IEEE Trans. on Automatic Control, 47(2):253-270, 2002.

[5] E. Fridman and U. Shaked. Delay dependent stability and $h_{\infty}$ control: constant and time-varying delays. International Journal of Control, 76(1):48-60, 2003.

[6] E. Fridman, U. Shaked, and K. Liu. New conditions for delayderivative-dependent stability. International Journal of Control, 45(11):2723-2727, 2009.

[7] H. Fujioka. Stability analysis of systems with aperiodic sample-andhold devices. Automatica, 45(3):771-775, 2009.

[8] K. Gu, V.-L. Kharitonov, and J. Chen. Stability of time-delay systems. Birkhauser, 2003.

[9] Y. He, Q. G. Wang, C. Lin, and M. Wu. Delay-range-dependent stability for systems with time-varying delay. Automatica, 43:371376, 2007.

[10] Y. He, Q. G. Wang, L. Xie, and C. Lin. Further improvement of free-weighting matrices technique for systems with time-varying delay. IEEE Trans. on Automat. Control, 52:293-299, February 2007.

[11] X. Jiang and Q.L. Han. Delay-dependent robust stability for uncertain linear systems with interval time-varying delay. Automatica, 42(6):1059-1065, 2006.

[12] C.Y. Kao and A. Rantzer. Stability analysis of systems with uncertain time-varying delays. Automatica, 43(6):959 - 970, 2007.

[13] J.H. Kim. Note on stability of linear systems with time-varying delay. Automatica, 47(9):2118-2121, 2011.

[14] K. Liu and E. Fridman. Wirtinger's inequality and lyapunov-based sampled-data stabilization. Automatica, 48(1):102108, 2012.

[15] L. Mirkin. Some remarks on the use of time-varying delay to model sample-and-hold circuits. IEEE Trans. on Automatic Control, 52(6):1009-1112, 2007.

[16] S.-I. Niculescu. Delay Effects on Stability. A Robust Control Approach. Springer-Verlag, 2001.

[17] P.G. Park, J. W. Ko, and C. Jeong. Reciprocally convex approach to stability of systems with time-varying delays. Automatica, 47:235238, 2011.

[18] J.-P. Richard. Time delay systems: an overview of some recent advances and open problems. Automatica, 39:1667-1694, 2003.

[19] A. Seuret and F. Gouaisbaut. On the use of the wirtinger's inequalities for time-delay systems. In Proc. of the $10^{\text {th }}$ IFAC Workshop on Time Delay Systems (IFAC TDS'12), Boston, MA, USA, 2012.

[20] A. Seuret and F. Gouaisbaut. Integral inequality for time-varying delay systems. to appear in the European Control Conference, 2013.

[21] H. Shao. New delay-dependent stability criteria for systems with interval delay. Automatica, 45(3):744 - 749, 2009.

[22] R. Sipahi, S. Niculescu, C.T. Abdallah, W. Michiels, and K. Gu. Stability and stabilization of systems with time delay. Control Systems, IEEE, 31(1):38 -65, feb. 2011.

[23] J. Sun, G.P. Liu, J. Chen, and D. Rees. Improved delay-rangedependent stability criteria for linear systems with time-varying delays. Automatica, 46(2):466 - 470, 2010. 